\title{
THE DEEP LUMINOSITY FUNCTION OF THE GLOBULAR CLUSTER M30 ${ }^{1}$
}

\author{
Giampaolo Piotto, ${ }^{2}$ Ivan R. King, ${ }^{3}$ Massimo Capaccioli, ${ }^{4}$ Sergio Ortolani, ${ }^{4}$ ANd S. Djorgovski ${ }^{5}$ \\ Received 1989 March 24; accepted 1989 August 10
}

\begin{abstract}
This paper is aimed at determining the mass function in the low-metallicity, post-core collapse globular cluster NGC $7099=\mathrm{M} 30$, and analyzing its radial trend. New deep CCD photometry is presented in two fields centered at $r=2.9$ and $4: 4$ from the center, respectively.

We confirm that a good representation of the color-magnitude diagrams is obtained by oxygen-enhanced isochrones (as used by Capaccioli, Ortolani, and Piotto). The best fit yields $(m-M)_{V}=14.65, E(B-V)=0.05$, and an age of $16 \mathrm{Gyr}$.

Luminosity functions down to $V \simeq 25\left(M_{V} \simeq 10.3\right)$ are constructed and carefully corrected for contamination by field objects and for incompleteness (see Appendix). Comparison with standard theoretical luminosity functions shows that the mass function, modeled with a power law, has an index $x \simeq 1$, at variance with the $x \simeq 0$ found by Richer, Fahlman, and VandenBerg for a field centered at $r=3.4$. An even steeper slope is given by the photometry in an outer field of M30 studied with the $4 \mathrm{~m}$ CTIO CCD camera (Piotto et al.).

All together, our data agree with the trend of $x$ found by Pryor, Smith, and McClure for a multimass KingMichie model (with or without velocity anisotropy), yielding a global slope $x_{0} \sim 0.7$. Mass segregation is implied, though marginally, given the errors and the small variation of $x$ expected on theoretical grounds in the radial range explored by our observations.

M30 is found to deviate strongly from the mass-function slope versus metallicity relation (McClure et al.); its mass function is as flat as that of intermediate-metallicity clusters.
\end{abstract}

Subject headings: clusters: globular - luminosity function - stars: abundances - stars: evolution

\section{INTRODUCTION}

NGC $7099=\mathrm{M} 30(\mathrm{C} 2137-234)$ is one of the most metalpoor globular clusters in the Galaxy $([\mathrm{Fe} / \mathrm{H}]=-2.19$; Webbink 1985). Since Dickens' (1972) early photographic photometry, its stellar content has been subjected to several investigations (see Piotto et al. 1987; Capaccioli, Ortolani, and Piotto 1987; Piotto, King, and Djorgovski 1988; Richer, Fahlman, and VandenBerg 1988, hereafter RFV, for references). RFV have given a luminosity function (LF) of M30 based on CCD photometry of a field at a mean distance $r=3.4$ from the cluster center. Comparing their LF with an unpublished LF from Bolte's (1987) photometry of a field at $r=6^{\prime}$, these authors conclude that the cluster shows evidence of a strong mass segregation in the low-mass stars, presumably acquired through dynamical relaxation. At the same time, they emphasize that the segregation is much greater than predicted by multimass King-Michie models (Pryor, Smith, and McClure 1986). The RFV results have such an importance for our understanding of the dynamical evolution of globular clusters as to deserve further verification. To this end, we planned our own CCD observations of M30, taking care to interlace new fields with those previously mapped by RFV and Bolte.

Deep exposures of two fields in M30 (F1 and F2; Table 1)

\footnotetext{
${ }^{1}$ Based on observations collected at the European Southern Observatory, La Silla, and at Cerro Tololo Inter-American Observatory. G. P. is a Visiting Astronomer at the European Southern Observatory, La Silla, Chile. S. D. is a Visiting Astronomer at Cerro Tololo Inter-American Observatory, which is operated by the Association of Universities for Research in Astronomy, Inc., under contract with the National Science Foundation.

${ }^{2}$ Dipartimento di Astronomia, Università di Padova, Italy.

${ }^{3}$ Berkeley Astronomy Department, University of California.

${ }^{4}$ Osservatorio Astronomico, Padova, Italy.

5 Division of Physics, Mathematics, and Astronomy, California Institute of Technology.
}

were secured at the Cassegrain focus of the $2.2 \mathrm{~m} \mathrm{ESO/MPI}$ telescope equipped with the RCA CCD \#5 detector (pixel scale $=0$ '.35, for an effective field size of $1^{\prime} .9 \times 3.0$ ). Field F1 is centered at $r=4.4$ northeast of the cluster center. Field F2 $(r=2.9$ north) is a 1.9 $\times 1.9$ subframe of a CCD picture; the part of the frame closer to the center, being too crowded for resolution, was rejected. This material is supplemented by very deep photometry of an outer field ( $r=7^{\prime}$ west) from CCD images taken at the prime focus of the $4 \mathrm{~m}$ CTIO telescope (Table 1). The radial coverage of the centers of our frames $(2.9-7.0)$ is thus larger than that available to RFV (3.4-6'0).

This paper is structured as follows. Photometry and colormagnitude (c-m) diagrams of fields $F 1$ and F2 are presented in $\S$ II. The discussion of these data is limited to their use for the derivation and analysis of the corresponding luminosity functions ( $\S$ III); a full analysis of contamination by foreground/ background objects and of completeness corrections is moved to the Appendix in order to enhance the continuity of the astrophysical discussion. The mass function (MF) is compared in $\S$ IV with the results of RFV and with the multimass KingMichie models; there we will attempt to infer the global mass function from the mass functions derived at various distances from the center of M30.

\section{PHOTOMETRY AND COLOR-MAGNITUDE DIAGRAM}

Preprocessing of the ESO frames has been performed with VISTA, and all the photometric reductions have been performed with DAOPHOT, following the precepts outlined by Stetson (1987). Transformations of $b$ and $v$ instrumental magnitudes to the $B$ and $V$ Landolt (1973) systems,

$$
\begin{gathered}
B=b+(0.143 \pm 0.011)(B-V)+(\text { const } \pm 0.009), \\
V=v+(0.015 \pm 0.006)(B-V)+(\text { const } \pm 0.004),
\end{gathered}
$$


TABLE 1

M30: CCD MATERIAL

\begin{tabular}{|c|c|c|c|}
\hline Region & Center & Band & $\begin{array}{c}\text { Exposures } \\
\text { (s) }\end{array}$ \\
\hline F1 (ESO) $\ldots \ldots \ldots$ & $1^{\prime} .1 \mathrm{E} ; 4^{\prime} .1 \mathrm{~N}$ & $\begin{array}{l}B \\
V\end{array}$ & $\begin{array}{l}2 \times 2400 \\
2 \times 1200\end{array}$ \\
\hline F2 (ESO) $\ldots \ldots \ldots$ & $0.5 \mathrm{~W} ; 2.3 \mathrm{~N}$ & $\begin{array}{l}B \\
V\end{array}$ & $\begin{array}{l}2 \times 2400 \\
2 \times 1200\end{array}$ \\
\hline CTIO ............ & $7.0 \mathrm{~W} ; 0^{\prime} .0 \mathrm{~N}$ & $\begin{array}{l}B \\
B \\
B \\
B \\
V \\
V \\
V\end{array}$ & $\begin{array}{r}1 \times 400 \\
19 \times 500 \\
1 \times 600 \\
2 \times 700 \\
12 \times 300 \\
8 \times 400 \\
6 \times 500\end{array}$ \\
\hline
\end{tabular}

were derived by means of 11 Landolt (1983) standard stars, measured with the aperture photometry routine of DAOPHOT. Since standard stars and M30 frames had approximately the same seeing, the integration area was kept constant. The brightest, not saturated, and most isolated cluster stars were used to tie together the aperture photometry instrumental magnitudes and those given by the multiple PSF fitting routine; this matching introduces zero-point uncertainties of $\pm 0.003 \mathrm{mag}$ in the $V$ band, and $\pm 0.005 \mathrm{mag}$ in the $B$ band, which must be combined with those of equation (1). The final uncertainty of our photometric zero point is then $\pm 0.005 \mathrm{mag}$ for the $V$ band and $\pm 0.010 \mathrm{mag}$ for the $B$ band, corresponding to an error of $\pm 0.011 \mathrm{mag}$ in the $B-V$ color. These figures are probably lower limits, because they do not take into account the differences in metallicity and luminosity class between the standards and the M30 stars (touched on peripherally by Weisz and Bell 1989 and under study in a systematic way by some of us [King et al. 1989]).

The c-m diagrams for the two ESO fields are reproduced in Figures $1 a$ and $1 b$. They include only stars carefully selected for quality of measurement. First, each star was visually inspected in a zoomed display to eliminate galaxies, faint stars on the edge of bright images, and stars that impinged on bad CCD columns. Then a further selection chose stars whose $B$ and $V$ positions on registered frames agree within 1 pixel $(0.35)$ and which have $0<\chi<1.5$ and $-0.2<$ SHARP $<0.2$
TABLE 2

M30 Main-Sequence Fiducial Points AND WIDTHS

\begin{tabular}{ccccc}
\hline \hline$V$ & $B-V$ & $\sigma_{\text {obs }}$ & $\sigma_{\text {DAO }}$ & $\sigma_{\text {art }}$ \\
\hline $17.95 \ldots \ldots$ & 0.501 & 0.002 & 0.001 & $\ldots$ \\
$18.25 \ldots \ldots$ & 0.422 & 0.002 & 0.001 & $\ldots$ \\
$18.55 \ldots \ldots$ & 0.401 & 0.003 & 0.006 & 0.002 \\
$18.85 \ldots \ldots$ & 0.398 & 0.003 & 0.007 & 0.003 \\
$19.15 \ldots \ldots$ & 0.416 & 0.009 & 0.007 & 0.008 \\
$19.45 \ldots \ldots$ & 0.442 & 0.020 & 0.007 & 0.006 \\
$19.75 \ldots \ldots$ & 0.454 & 0.014 & 0.009 & 0.010 \\
$20.05 \ldots \ldots$ & 0.477 & 0.019 & 0.009 & 0.009 \\
$20.35 \ldots \ldots$ & 0.520 & 0.022 & 0.011 & $\ldots$ \\
$20.65 \ldots \ldots$ & 0.578 & 0.022 & 0.013 & $\ldots$ \\
$20.95 \ldots \ldots$ & 0.625 & 0.042 & 0.016 & $\ldots$ \\
$21.25 \ldots \ldots$ & 0.682 & 0.042 & 0.020 & $\ldots$ \\
$21.55 \ldots \ldots$ & 0.773 & 0.055 & 0.023 & $\ldots$ \\
$21.85 \ldots \ldots$ & 0.795 & 0.073 & 0.032 & $\ldots$ \\
$22.15 \ldots \ldots$ & 0.856 & 0.098 & 0.038 & $\ldots$ \\
$22.45 \ldots \ldots$ & 0.924 & 0.132 & 0.051 & 0.125 \\
$22.75 \ldots \ldots$ & 1.051 & 0.210 & 0.071 & 0.237 \\
$23.05 \ldots \ldots$ & 0.981 & 0.204 & 0.078 & 0.215 \\
$23.35 \ldots \ldots$ & 1.081 & 0.224 & 0.099 & 0.233 \\
\hline
\end{tabular}

(DAOPHOT parameters; see Stetson 1987), and a photometric error, as given by DAOPHOT, smaller than 3 times the mean error for their magnitude. All together, these criteria rejected $30 \%$ of the stars detected in F1 and $46 \%$ of those detected in $\mathrm{F} 2$, which were removed when constructing the $\mathrm{c}-\mathrm{m}$ diagrams.

Figure $1 a$ shows some evidence that the scatter is larger on the red side of the main sequence (MS). Stetson and Harris (1988) suggest that a similar feature in the c-m diagram of M92 could be due to overlapping star images. An alternative possibility, which we favor, is binaries. The first two authors of this paper will discuss this question in a separate paper.

The fiducial points of Table 2, derived from the "clean" sample of 475 and 329 stars for regions F1 and F2, respectively, are the mode of the $B-V$ marginal distribution for $0.3 \mathrm{mag}$ wide bins in $V$. These fiducial points are compared with those from other CCD sources in Figure 2. There is good agreement with RFV down to their limit $V=22.3$, although our sequence is $\sim 0.01 \mathrm{mag}$ bluer, on average. (We would not be surprised to find discrepancies up to $\pm 0.03 \mathrm{mag}$ among different "good"

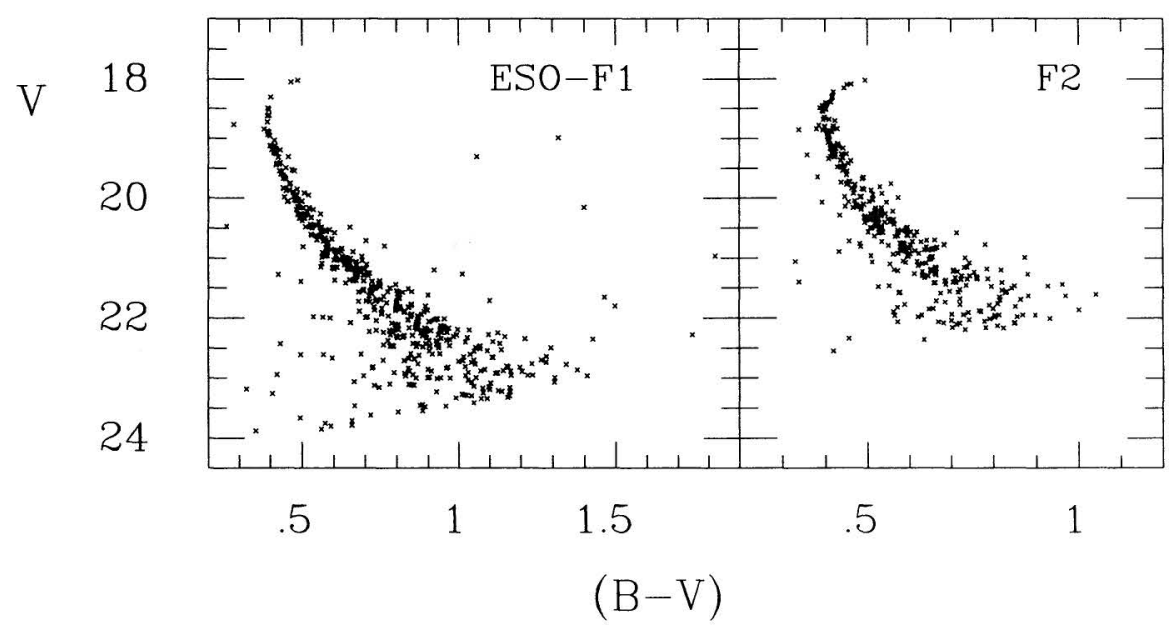

Fig. 1.-Color-magnitude diagrams for the two ESO regions of M30 at $r=2.9$ (F2) and $r=4.4$ (F1) 


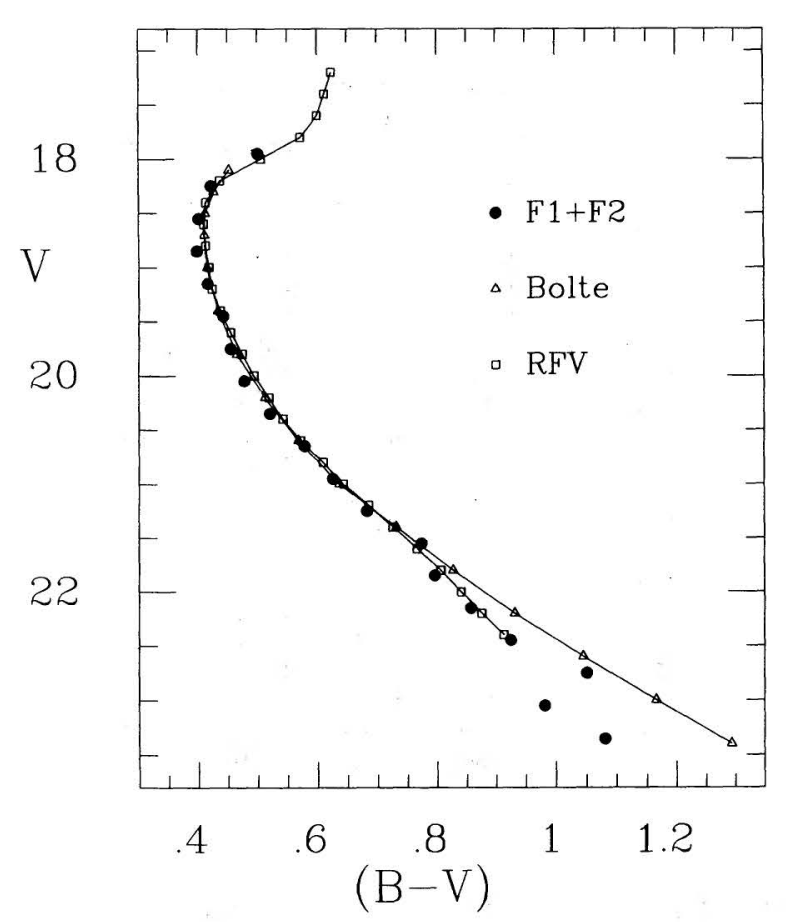

FIG. 2.-Color-magnitude diagram: fiducial points for the combined photometry in our two fields compared with Bolte and RFV data. Note the good agreement with RFV (although our data are systematically bluer, possibly because of the different method adopted to compute the representative points), and the increasingly larger disagreement with Bolte at the faint end.

photometries.) In addition to a zero-point shift, this systematic difference is possibly due to the fact that the RFV fiducials are mean points, not modes as they are here (a fact that might support the reality of the skewness about the mean MS reported above). The fiducial sequence published by Bolte is increasingly redder than RFV's and ours for $V>21.5$, the difference reaching $\Delta(B-V) \simeq 0.2$ at $V=23.4$.

It seems instructive to judge the observed MS width, $\sigma_{\text {obs }}$ (Table 2), against the broadening which is expected from the photometric errors only. It is known that DAOPHOT tends to underestimate errors in magnitudes (Stetson and Harris 1988). The effect is made evident at the faintest levels by the comparison of $\sigma_{\mathrm{DAO}}(B-V)$ (calculated using DAOPHOT errors on $B$ and $V$ photometry) with the same quantity, $\sigma_{\text {art }}$, provided by the artificial star experiments (Table 2 and the Appendix). Therefore, in order to estimate an upper limit to the intrinsic width, we shall take out the instrumental broadening. In practice, we assume $\sigma_{\mathrm{art}}$ to represent the "instrumental width," and we compute the intrinsic width $\Delta(B-V)$ as the mean value of $\left(\sigma_{\text {obs }}^{2}-\sigma_{\text {art }}^{2}\right)^{1 / 2}$, over three consecutive magnitude bins. The VandenBerg and Bell (1985) models predict $\Delta(B-V) / \Delta[M /$ $H] \sim 0.05$ around the turnoff. Considering the interval $18.4<V<20.2$, and assuming that the intrinsic width of the MS $[\langle\Delta(B-V)\rangle=0.009]$ is due to measurement errors and chemical inhomogeneity only (though there may be other causes, such as binaries or differential reddening), we can conclude that $\Delta[M / H]<0.18 \pm 0.06$, in agreement with the $\Delta[M / H]<0.20$ found by RFV.

Since the cluster parameters have been extensively discussed by Bolte and by RFV, suggesting ages in the range 14-18 Gyr, we have opted to use a $16 \mathrm{Gyr}$, oxygen-enhanced isochrone

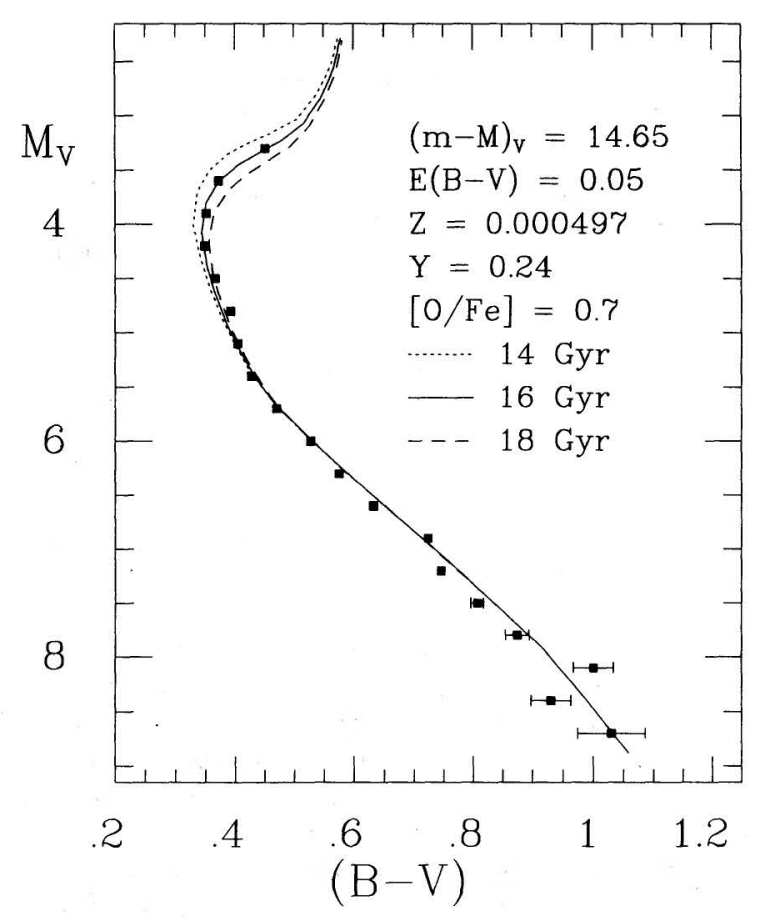

Fig. 3.-Combined photometry in the $\mathrm{F} 1$ and $\mathrm{F} 2$ regions of $\mathrm{M} 30$ (filled squares) compared with oxygen-enhanced theoretical isochrones from McClure et al. (1987b). The best fit (solid line) can be used to estimate both the distance modulus and the age of the cluster.

from McClure et al. (1987b) in the following analysis. By matching our data to this isochrone, we get $(m-M)_{V}=14.65$ and $E(B-V)=0.05$ (values within the range found by Bolte and RFV), leading to the rather good fit shown in Figure 3. We want to stress that small differences in distance and age are of minimal consequence for the conclusions to be reached in the following sections.

\section{THE LUMINOSITY FUNCTION}

The luminosity function in each ESO region has been obtained from the corresponding photometry. Two different approaches have been followed:

1. $\mathrm{LF}_{B V}$ is the luminosity function containing only stars which have been identified in both the $B$ and the $V$ frames and which have relative positions coinciding within 1 pixel. In contrast with $\S \mathrm{II}$, no selection based on DAOPHOT parameters has been applied; that selection would inevitably penalize the faint end of the luminosity function, thus introducing an unwanted bias. We took advantage of the color-magnitude diagram to reject objects whose color indicated that they must be field stars. To do this, we drew lines, by hand, on either side of the main sequence, at a conservative distance of about 5 or 6 $\sigma$ in $B-V$ from the fiducial points. Fainter than $V=22.5$, the color spread becomes too large for this procedure; there we extrapolated the number of field objects from brighter magnitudes, on a scale of $\log \Phi$ versus $V$, as explained in $\S I$ of the Appendix. The advantage of the two-color approach is that it favors the rejection of background/foreground objects and of objects that are really just noise peaks. However, the resulting $\mathrm{LF}$ is constrained by the limiting magnitude in the $B$ band.

2. $\mathrm{LF}_{\boldsymbol{V}}$ uses the entire set of stars identified in the $V$ frame alone, i.e., all the information inherent in our material. 
TABLE 3

LUMINOSITY FUNCTIONS

\begin{tabular}{crrrr}
\hline \hline $\begin{array}{c}V \\
(1)\end{array}$ & $\begin{array}{c}\boldsymbol{\Phi}_{0} \\
(2)\end{array}$ & $\begin{array}{c}100 / \gamma \\
(3)\end{array}$ & $\begin{array}{c}\boldsymbol{\Phi}_{\text {ficld }} \\
(4)\end{array}$ & $\begin{array}{r}\Phi_{c} \\
(5)\end{array}$ \\
\hline \multicolumn{5}{c}{ A. $\mathbf{L F}_{\boldsymbol{B} \boldsymbol{V}}:$ Region F1 } \\
\hline $17.9 \ldots \ldots$ & 4 & 100 & 0 & 4 \\
$18.4 \ldots \ldots$ & 11 & 100 & 0 & 11 \\
$18.9 \ldots \ldots$ & 20 & 100 & 2 & 18 \\
$19.4 \ldots \ldots$ & 34 & 100 & 4 & 30 \\
$19.9 \ldots \ldots$ & 41 & 100 & 0 & 41 \\
$20.4 \ldots \ldots$ & 59 & 100 & 7 & 52 \\
$20.9 \ldots \ldots$ & 53 & 100 & 8 & 45 \\
$21.4 \ldots \ldots$ & 71 & 97 & 7 & 66 \\
$21.9 \ldots \ldots$ & 84 & 93 & 10 & 80 \\
$22.4 \ldots \ldots$ & 104 & 89 & 14 & 103 \\
$22.9 \ldots \ldots$ & 97 & 71 & 17 & 119 \\
$23.4 \ldots \ldots$ & 72 & 30 & 22 & 218 \\
$23.9 \ldots \ldots$ & 37 & 12 & 27 & 281 \\
\hline
\end{tabular}

B. $\mathrm{LF}_{\boldsymbol{V}}$ : Region $\mathrm{F}$

\begin{tabular}{rrrrr}
\hline $17.9 \ldots \ldots$ & 5 & 100 & 0 & 5 \\
$18.4 \ldots \ldots$ & 11 & 100 & 0 & 11 \\
$18.9 \ldots \ldots$ & 22 & 100 & 2 & 20 \\
$19.4 \ldots \ldots$ & 37 & 100 & 4 & 33 \\
$19.9 \ldots \ldots$ & 43 & 100 & 0 & 43 \\
$20.4 \ldots \ldots$ & 62 & 100 & 7 & 55 \\
$20.9 \ldots \ldots$ & 59 & 100 & 8 & 51 \\
$21.4 \ldots \ldots$ & 84 & 100 & 7 & 77 \\
$21.9 \ldots \ldots$ & 94 & 100 & 10 & 84 \\
$22.4 \ldots \ldots$ & 134 & 100 & 14 & 120 \\
$22.9 \ldots \ldots$ & 168 & 98 & 17 & 15 \\
$23.4 \ldots \ldots$ & 201 & 96 & 22 & 188 \\
$23.9 \ldots \ldots$ & 258 & 89 & 28 & 263 \\
$24.4 \ldots \ldots$ & 267 & 55 & 34 & 452 \\
$24.9 \ldots \ldots$ & 151 & 25 & 42 & 562 \\
\hline
\end{tabular}

C. $\mathrm{LF}_{B V}$ : Region $\mathrm{F} 2$

$\begin{array}{rrrrr}17.9 \ldots \ldots & 6 & 100 & 0 & 6 \\ 18.4 \ldots \ldots & 16 & 100 & 1 & 15 \\ 18.9 \ldots \ldots & 31 & 100 & 1 & 30 \\ 19.4 \ldots \ldots & 46 & 100 & 2 & 44 \\ 19.9 \ldots \ldots & 55 & 100 & 2 & 53 \\ 20.4 \ldots \ldots & 72 & 99 & 3 & 70 \\ 20.9 \ldots \ldots & 75 & 94 & 2 & 78 \\ 21.4 \ldots \ldots & 87 & 86 & 3 & 98 \\ 21.9 \ldots \ldots & 84 & 69 & 3 & 119 \\ 22.4 \ldots \ldots & 73 & 44 & 5 & 161\end{array}$

D. $\mathbf{L F}_{\boldsymbol{V}}$ : Region $\mathbf{F} 2$

\begin{tabular}{rrrrr}
\hline $17.9 \ldots \ldots$ & 6 & 100 & 0 & 6 \\
$18.4 \ldots \ldots$ & 16 & 100 & 1 & 15 \\
$18.9 \ldots \ldots$ & 34 & 100 & 1 & 33 \\
$19.4 \ldots \ldots$ & 53 & 100 & 2 & 51 \\
$19.9 \ldots \ldots$ & 60 & 100 & 2 & 58 \\
$20.4 \ldots \ldots$ & 83 & 100 & 3 & 80 \\
$20.9 \ldots \ldots$ & 90 & 98 & 2 & 90 \\
$21.4 \ldots \ldots$ & 101 & 93 & 3 & 106 \\
$21.9 \ldots \ldots$ & 104 & 88 & 3 & 115 \\
$22.4 \ldots \ldots$ & 110 & 76 & 5 & 140 \\
$22.9 \ldots \ldots$ & 92 & 58 & 6 & 153 \\
$23.4 \ldots \ldots$ & 89 & 38 & 6 & 228 \\
\hline & E. LF & LV CTIO & & \\
& & & \\
\hline $18.4 \ldots \ldots$ & 9 & 100 & 2 & 7 \\
$18.9 \ldots \ldots$ & 14 & 100 & 3 & 11 \\
$19.4 \ldots \ldots$ & 16 & 100 & 3 & 13 \\
$19.9 \ldots \ldots$ & 19 & 100 & 4 & 15 \\
$20.4 \ldots \ldots$ & 31 & 100 & 5 & 26 \\
$20.9 \ldots \ldots$ & 31 & 100 & 5 & 26 \\
$21.4 \ldots \ldots$ & 36 & 100 & 7 & 29 \\
$21.9 \ldots \ldots$ & 65 & 100 & 8 & 57 \\
$22.4 \ldots \ldots$ & 48 & 95 & 10 & 40 \\
$22.9 \ldots \ldots$ & 63 & 94 & 12 & 54 \\
$23.4 \ldots \ldots$ & 99 & 89 & 15 & 94 \\
$23.9 \ldots \ldots$ & 149 & 80 & 25 & 156 \\
\hline & & & &
\end{tabular}

Completeness and contamination corrections are presented and discussed in the Appendix; independent estimates of the completeness corrections were made for each of the above LFs.

The luminosity functions, $\Phi$, are presented in Table 3 . Column (2) gives the number $\Phi_{0}$ of objects actually counted within each bin (observed LF), and columns (3) and (4) list the completeness (as a percentage: $100 \gamma^{-1}$ ) and the number of field objects, $\Phi_{\text {field }}$. Once applied to the observed total number of cluster plus field stars in each magnitude bin, the correction factor $\gamma(\geq 1)$ removes the incompleteness. It has been estimated through artificial star experiments (§ II of the Appendix).

The completeness-corrected and field object-cleaned numbers of M30 stars per bin, $\Phi_{c}$, are listed in column (5). Both the observed and the corrected LFs are plotted in Figure 4. (Note that the corrected numbers can be less than the observed, on account of the subtraction of field stars.) The error bars, calculated assuming a Poisson distribution, are somewhat underestimated, because they do not include the errors in the completeness factor and in the number $\Phi_{\text {field }}$ of the field objects. As expected, the deepest LF comes from the $V$ frames of region F1. Crowding experiments in this field indicate that the sample is complete down to $V=22.65$ and that more than $90 \%$ of all stars have been found at all $V \lesssim 24$.

\section{THE MASS FUNCTION}

We adopt $(m-M)_{V}=14.65 \mathrm{mag}$ from a match of the photometry to the $16 \mathrm{Gyr}$ isochrone; however, our conclusions would not change if isochrones for an age differing by $\pm 2 \mathrm{Gyr}$ were adopted or if we varied the distance within $\pm 10 \%$. Numerical experiments have shown that if we adopt a distance modulus of 14.85, the resulting mass function has a slope $\sim 0.2$ units smaller than using $m-M=14.65$.

In Figure 5, the corrected luminosity functions, $\Phi_{c}$, for the two ESO regions are compared with the theoretical predictions based on a power-law mass function (MF), $d \xi=$ $\xi_{0} m^{-x} d \log m$, with $x=0.0,1.0,2.0$. The mass-luminosity relation has been interpolated from the values tabulated by McClure et al. (1987b) for a coeval stellar population with age of $16 \mathrm{Gyr}$, helium abundance of $Y=0.24$, and metallicity of $Z=4.97 \times 10^{-4}$. The filled dots represent $L_{V}$ for region $\mathrm{F} 1$; the open triangles refer to $F 2$. Even from visual inspection of this figure, it is apparent that the power-law index of the MF must be in the range $0.8<x<1.2$.

The filled squares in Figure 5 reproduce the LF published by RFV for their field at 3'.4 from the center; their limiting magnitude, $V \sim 23.0$, is almost 2 mag brighter than our deepest field. It is clear from the figure that the RFV conclusion that the mass function in M30 is flat $(x \simeq 0)$ rests mostly on the value of $\Phi$ within the bin $22 \leq V \leq 23$ (or $\left\langle M_{V}\right\rangle=7.85$ ), where their completeness was quite low $\left(\gamma^{-1}=0.34\right)$. Consequently, their suggestion that $x$ is near zero is not robust. By contrast, the completeness of our $V$ photometry in region $\mathrm{F} 1$ is still $100 \%$ at the same level as the limiting magnitude of the RFV study.

Figure 5 plots also the LF for an outer M30 field $\left(r \sim 7^{\prime}\right)$ covered by CTIO CCD material (a full account will be given elsewhere; Piotto, King, and Djorgovski 1989). This field is much less crowded than those of the ESO, and its photometry is much deeper; however, the lack of a comparison field hampers, for now, the exploitation of the entire photometric range. Again, the LF appears to be even steeper than the curve predicted by an $x=1.0$ power-law mass function; however, the 


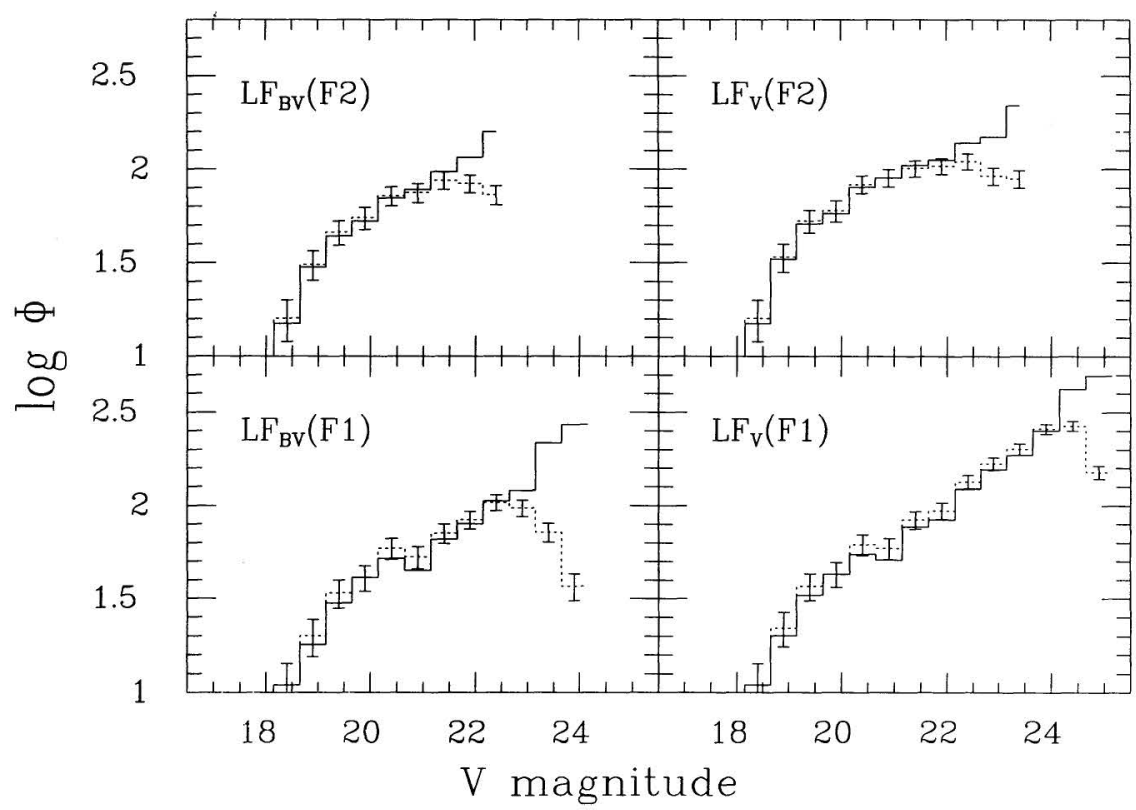

Fig. 4.-Dotted lines represent the observed luminosity functions (see text), which turn into solid lines once corrections for field contamination and incompleteness are applied. Error bars indicate Poisson uncertainties.

noise is larger than in the ESO data because of the relatively smaller number of stars in this outer field.

In the following, we present an attempt to measure the dependence of $x$ on the mean distance $\langle r\rangle$ from the cluster center. This strategy has the disadvantage that we are dependent on a theoretical transformation from luminosities to

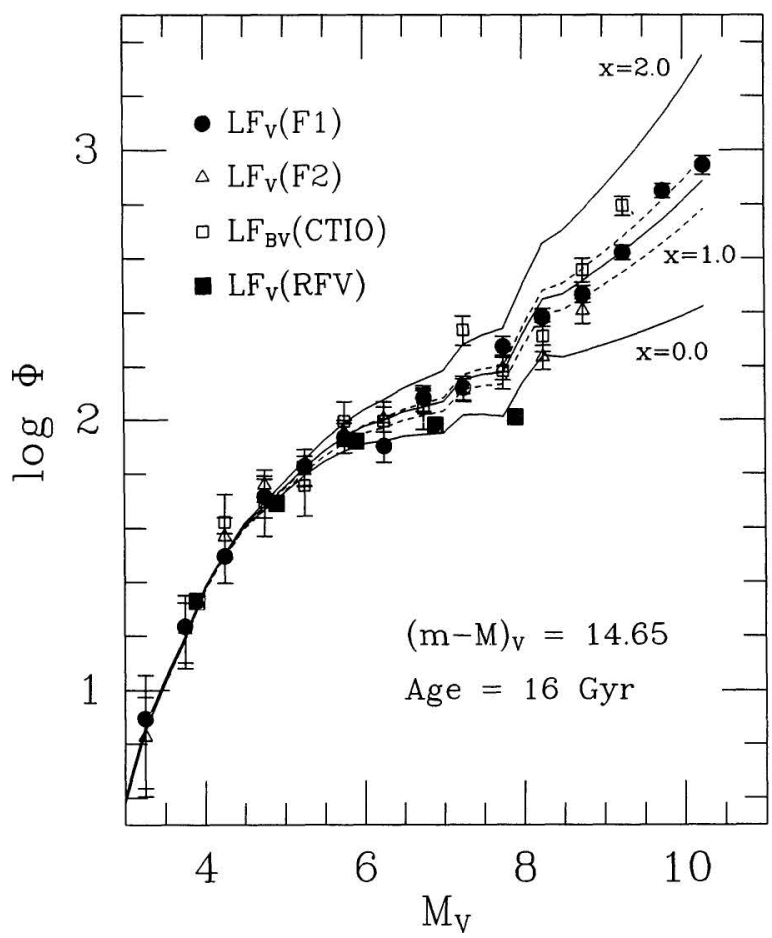

Fig. 5.-Luminosity functions of four M30 regions plotted against the theoretical curves (solid lines) derived with the McClure et al. (1987b) massluminosity relation assuming a power-law mass function and three values of the power index $x$. Dotted lines $(x=0.8$ and 1.2$)$ show the uncertainty in $x$. Range and trend of the RFV data are shown by the filled squares. masses, and it requires a statistically undesirable transfer from the observational to the theoretical domain, but it has the distinct advantage that we can see how well a power law actually fits. In Figure 6, we plot the MF computed from the LFs using the mass-luminosity relation of McClure et al. (1987b). For each ESO region, we present the MF coming both from the $V$ photometry alone $\left(\mathrm{LF}_{V}\right)$ and from the c-m diagram $\left(\mathrm{LF}_{B V}\right)$; the completeness corrections are estimated independently in the two cases, so that the spread in $x$ is indicative of the accuracy of our result. We also present the MF from the $\mathrm{c}-\mathrm{m}$ diagram for CTIO. A straight line has been fitted to the data weighted according to their Poisson errors, ignoring those with a completeness $\gamma^{-1}<0.5$. The resulting slopes and their formal errors (Table 4) are $x=0.8 \pm 0.4$ for field F2 $(\langle r\rangle=2$ '.7), $x=1.1 \pm 0.2$ for $\mathrm{F} 1(\langle r\rangle=3.8)$, and $x=1.4 \pm 0.2$ for the CTIO field at $\langle r\rangle=6.2$. The quoted $\langle r\rangle$ values refer to distances from the cluster center weighted over the frames according to surface brightness (modeled using a King (1962) formula with $r_{c}=0.25$ and $\left.r_{t}=15^{\prime} .8\right)$; these light barycenters have been computed also for the RFV ${ }^{6}$ and Bolte fields (Table 4). Note that, although the very center of this post-collapse core cluster is not well-fitted by the King formula and $r_{c}$ is consequently ill defined, this uncertainty has very little effect on the values of $\langle r\rangle$.

The slope of the MF for the outer CTIO field is only marginally consistent with the value of $x$ for the two inner ESO fields; it agrees better with that found by Bolte (reported by RFV) in his field at $\langle r\rangle=5^{\prime}$. This fact, reinforced by an inspection to the data of Table 4, could be interpreted as an effect of mass segregation: stars of different mass are distributed differently in a relaxed cluster. Pryor, Smith, and McClure (1986) have constructed multimass King-Michie models with power-law MFs in order to investigate the radial dependence of the observed slope $x$ in a relaxed cluster. The aim of this work was to evaluate the mass-segregation corrections needed in recovering

\footnotetext{
${ }^{6}$ Note that the orientation of the RFV frames is reported incorrectly; the long axis of their CCD image is actually at P.A. $\sim 18^{\circ}$.
} 


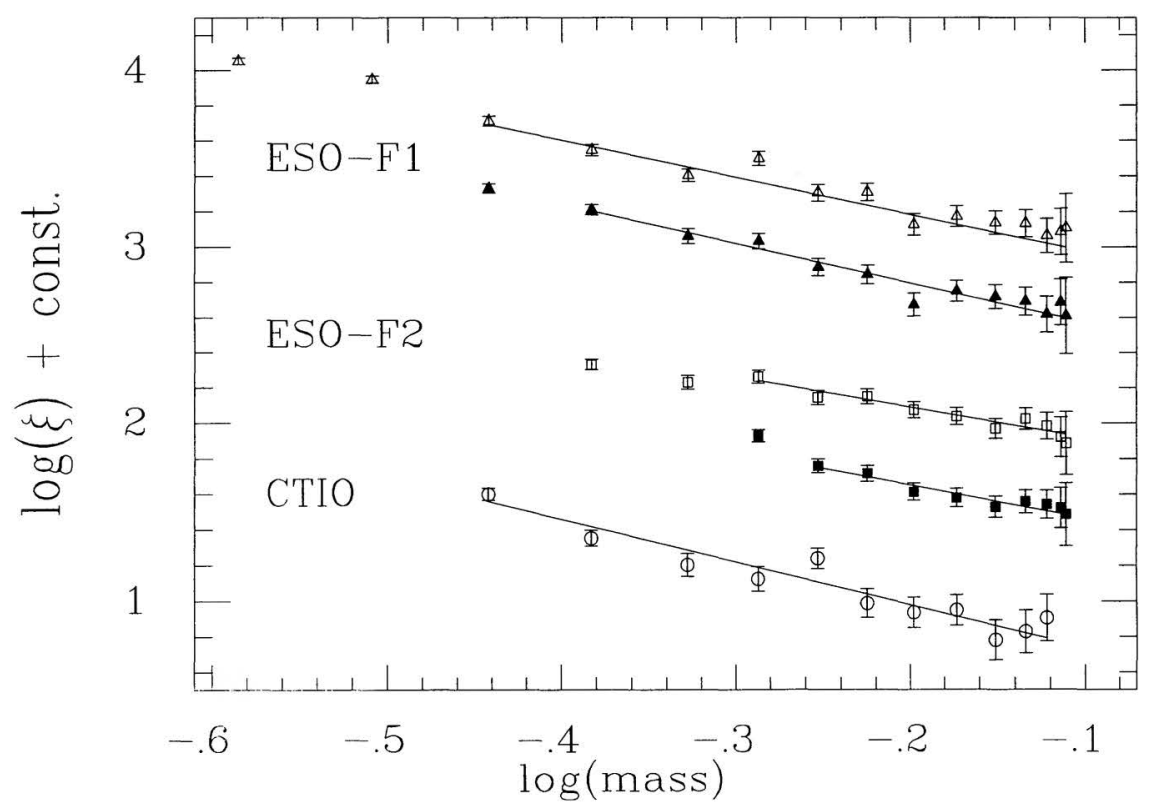

Fig. 6.-Mass functions for the ESO and CTIO fields: open symbols represent $L F_{Y}$ 's; filled symbols represent $L F_{B V}$ 's. The zero points of log $(\xi)$ have been altered to avoid overlapping. The shifts are (from the top to the bottom curve): $\Delta \log (\xi)=0.0,-0.4,-1.3,-1.7$, and -2.2 . Linear fits, shown as solid lines, have been limited to data with completeness factors better than $\gamma^{-1}=0.5$ (see also Table 4).

the global slope $x_{0}$ from that observed at a given $\langle r\rangle$ (measured in units of the core radius $r_{c}$ ). The models are computed for a set of concentration parameters $c=\log \left(r_{t} / r_{c}\right)$ (where $r_{t}$ is the tidal radius). They predict an increase of the local (observed) MF exponent above the global exponent $x_{0}$ at large distances from the center. The effect is larger in models with steeper MF and larger central concentration.

In order to apply the Pryor, Smith, and McClure (1986) grid of $x(r)$ to M30 models, we need to establish the value of the concentration parameter for this cluster, i.e., $r_{c}$ and $r_{t}$. The tidal radius is generally accepted to be $15^{\prime} .8$ (Peterson and King 1975). There is no agreement, however, in the literature about the value of the core radius: determinations range from $r_{c}=$ 4".4 (reported by Webbink 1985) to 15".4 (Cordoni and Aurière 1984). Even before M30 was found to be a post-core collapse cluster (Djorgovski and King 1984), it was noticed that its inner luminosity profile did not follow a single-mass King law (Cordoni and Aurière 1984). Under these conditions, the core radius loses its meaning. We may nevertheless find a value of $r_{\mathrm{c}}$ fitting the King formula to the light profile everywhere but in the core. Using all available sources of photometric and star-

TABLE 4

Slope of the Power-Law Mass Function

\begin{tabular}{ccccc}
\hline \multicolumn{1}{c}{ Region } & $\langle r\rangle$ & $1+x$ & $\sigma_{x}$ & $\begin{array}{c}\text { Limiting Mass } \\
\left(M_{\odot}\right)\end{array}$ \\
\hline F2: $\mathrm{LF}_{B V} \ldots \ldots \ldots \ldots \ldots$ & 2.7 & 1.88 & 0.42 & 0.558 \\
$\mathrm{~F} 2: \mathrm{LF}_{V} \ldots \ldots \ldots \ldots \ldots$ & 2.7 & 1.74 & 0.30 & 0.517 \\
$\mathrm{~F} 1: \mathrm{LF}_{B V} \ldots \ldots \ldots \ldots \ldots$ & 3.8 & 2.23 & 0.18 & 0.414 \\
F1: $\mathrm{LF}_{V} \ldots \ldots \ldots \ldots \ldots$ & 3.8 & 1.91 & 0.13 & 0.361 \\
$\mathrm{CTIO}: \mathrm{LF}_{B V} \ldots \ldots \ldots \ldots$ & 6.2 & 2.40 & 0.18 & 0.361 \\
RFV: $\mathrm{LF}_{V} \ldots \ldots \ldots \ldots$ & 2.6 & 1.0 & $\ldots$ & $\sim 0.5$ \\
Bolte $\ldots \ldots \ldots \ldots \ldots \ldots$ & 5.0 & 2.6 & $\ldots$ & $\ldots$ \\
\hline
\end{tabular}

count profiles (16 independent data sets out to $r=13$ '.2), we find $r_{c} \simeq 15^{\prime \prime}$.

We adopt a concentration parameter $c=1.8$ for M30. Since our conclusions are not strongly dependent upon the value of $c$, we can compare our determinations of $x$ with a Pryor, Smith, and McClure (1986) model having $c=1.9$. Figure 7 shows also the points pertaining to the fields of Bolte and RFV. The plot is different from the equivalent Figure 9 in RFV: these authors say they have adopted the core radius given by

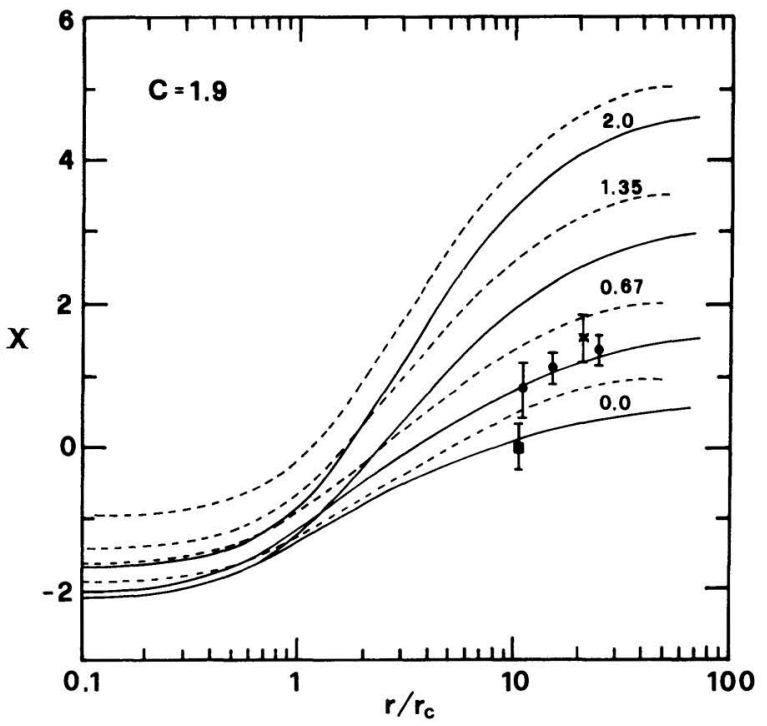

Fig. 7.-MF power indices (filled circles) for our three fields in M30 compared with the theoretical predictions by a multimass King-Michie model with concentration parameter $c=1.9$ : solid lines are for isotropic models, dashed lines are for anisotropic ones. Values from Bolte (cross) and RFV (square) are also plotted. The best estimate for the global power index is $x_{0} \simeq 0.7$. Trend of the data points is consistent with the occurrence of mass segregation. However, the effect to be measured is smaller than the errors. 
Webbink (1985; $r_{c}=4$ ".4), but they do not use this value consistently throughout their paper.

First of all, Figure 7 shows that the global value of the MF index must be close to $x_{0}=0.7$ (or even smaller, if anisotropy in the outer velocity field is taken into consideration).

Second, it gives the impression that the points match the model curves quite well. It must be clearly stated, however, that the expected change of $x$ is small over the range of $\langle r\rangle$ covered by our observations and that the data, with their error bars, are also consistent with no change at all (if we reject the RFV point). Note also that Pryor, Smith, and McClure (1986) comment on the accuracy and meaning of the results of their calculations, arguing that these might lead to an overestimate of the mass segregation.

More importantly, we note that not only is the value $x_{0} \simeq$ 0.7 inconsistent with the relation between metallicity and the steepness of the MF found by McClure et al. $(1986,1987 a)$; the values at all radii are inconsistent with their relation. According to that relation, metal-poor clusters should have mass functions steeper than metal-rich clusters (see Fig. 4 in Pryor, Smith, and McClure 1986, where both the observed and the corrected slopes are plotted). We find that the mass function of M30, a cluster as metal-poor as M15 $\left(x_{0}=1.4\right)$, is as flat as that of M3: $x_{0}=0.8$ (Pryor, Smith, and McClure 1986), $[\mathrm{Fe} / \mathrm{H}]=-1.5$.

Finally, the values of $x$ in Table 4 result from a fit of MFs in different mass ranges. Constraining the data to a common range $\left(0.56<m<0.77 M_{\odot}\right)$, we find $x=0.69 \pm 0.4$ for region $\mathrm{F} 2$ and $x=0.7 \pm 0.5$ for $\mathrm{F} 1$. The outer CTIO field retains a steeper slope $(x=1.4 \pm 0.7)$, but has a very high uncertainty owing to the small number of stars (184). But we should remember that a power law might not tell the full story. Although the statistics are admittedly weak, nearly all the sets of points in Figure 6 suggest a tendency for the MF to steepen for $\log m<0.4$ (corresponding to $M_{V}>9.0$ ). Such an effect has also been noticed in M13 by Drukier et al. (1988).

We should note one caution, however. In discussing M30 in the context of normal cluster dynamics, we should not lose sight of the fact that it is a post-collapse core cluster and might not in fact behave according to these models at all. After all, several of us (Piotto, King, and Djorgovski 1988) have already noted a strange and anti-intuitive color gradient in its central regions. Moreover, the post-collapse core cluster M15 has recently been shown (Seitzer, Peterson, and Cudworth 1989) to have a pattern of internal velocity dispersion that is not at all in accord with the nearly isothermal predictions of the KingMichie models. It is clear that collapsing and post-collapse clusters behave in ways that are still poorly understood; surprises are still possible-or even likely. Even with this reservation, however, we note that the difference between the values of $x$ in our regions, relatively far from the core, and the global value $x_{0}$ is rather insensitive to strange behavior in the core.

In summary, this work indicates that there is no strong gradient of $x\left(r / r_{c}\right)$ over the range covered by observations of M30, contrary to the claim of RFV. Within the uncertainties, our results are in satisfactory agreement with the model curves of Figure 7. Our results bear also on the correlation between MF power-law index and metallicity claimed by McClure et al. (1986); we find that the MF of the very metal-poor cluster M30 is almost as flat as those of intermediate-metallicity clusters. This conclusion concerning M30 is strengthened by the study of the metal-poor cluster NGC 6397 (Ortolani, Piotto, and Capaccioli 1989), which has $[\mathrm{Fe} / \mathrm{H}]=-2.02$ and $x_{0} \sim 0.2$. (The expected value from the correlation, as revised by Pryor, Smith, and McClure 1986, would be $x_{0} \sim 1$.2.) We are unable to establish whether the residual trend, if any, is real or whether, as suggested by Renzini and Fusi Pecci (1988), it is at least partly induced by the theoretical assumptions ("input physics to models and bolometric corrections").

We wish to thank R. Walterbos for his help in the computation of the foreground contamination with his revised version of the Bahcall and Soneira code and N. Metcalfe for communicating the Durham galaxy counts to us in advance of publication. G. P. acknowledges the hospitality of the Department of Astronomy, University of California at Berkeley, and the Fondazione Ing. A. Gini for partial support. The work done at Berkeley was supported by NASA Contract NAS5-28086. S. D. was supported in part by funds from Harvard University and California Institute of Technology.

\section{APPENDIX}

\section{CORRECTIONS TO THE LUMINOSITY FUNCTION}

\section{FOREGROUND/BACKGROUND CORRECTIONS}

The observed luminosity function must be corrected for the contamination by foreground and background objects. M30 is at high galactic latitude $\left(b=-46^{\circ} 8\right)$, so the number $N_{s}$ of foreground stars is expected to be small. Ratnatunga and Bahcall (1985) predict just under 3 stars per arcmin ${ }^{2}$ down to $V=23$. This prediction is confirmed by the results obtained by running a revised version (kindly made available to us by R. Walterbos) of the Bahcall and Soneira (1980) code (Table 5, col. [2]). Since no comparison field was available in the vicinity of $\mathrm{M} 30$, the number $N_{g}$ of background galaxies in the faint magnitude bins-larger than that of foreground stars as demonstrated just by visual inspection of the c-m diagram of region F1 - had to be estimated from the Metcalfe et al. (1989) galaxy counts. Unfortunately, however, these counts cannot be used directly in our case, since we do not know how many background galaxies have already been identified as such by the DAOPHOT finding routines (because of their size or their elongated shapes) and consequently rejected. Thus, we use only the property that the differential number counts of galaxies $\log N_{g}(m)$ are linear with the magnitude $m$ (Metcalfe et al. 1989), coupling it with our ability to count, in the original c-m diagram of region $\mathrm{F} 1$, the field objects in each magnitude bin brighter than $V \sim 22.5$. A linear interpolation of the logarithm frequency distribution (corrected for incompleteness) is then used to extrapolate the number of field objects to fainter bins (Table 5, col. [3]). The results for F1 are applied to F2 by simple rescaling to the appropriate area. For the CTIO region, we could estimate field stars down to $V=23.5$ from the CMD, and galaxies down to the same level by use of visual inspection of the image and the CMD. Note that the direct use of the Metcalfe et al. counts [a factor of 2 larger than the numbers coming from our extrapolation in the last three 
TABLE 5

FIELD OBJECTS IN $112^{\prime \prime} \times 179^{\prime \prime}$

\begin{tabular}{ccc}
\hline \hline$V$ & $N_{s}$ & $N_{g}$ \\
\hline $17.9 \ldots \ldots \ldots$ & 0.4 & 0 \\
$18.4 \ldots \ldots \ldots$ & 0.6 & 0 \\
$18.9 \ldots \ldots \ldots$ & 0.8 & 2 \\
$19.4 \ldots \ldots \ldots$ & 1.0 & 3 \\
$19.9 \ldots \ldots \ldots$ & 1.2 & 0 \\
$20.4 \ldots \ldots \ldots$ & 1.4 & 6 \\
$20.9 \ldots \ldots \ldots$ & 1.6 & 7 \\
$21.4 \ldots \ldots \ldots$ & 1.8 & 6 \\
$21.9 \ldots \ldots \ldots$ & 1.9 & 9 \\
$22.4 \ldots \ldots \ldots$. & 2.1 & 13 \\
$22.9 \ldots \ldots \ldots$ & 2.3 & 16 \\
$23.4 \ldots \ldots \ldots$ & 2.5 & 21 \\
$23.9 \ldots \ldots \ldots$ & 2.8 & 27 \\
$24.4 \ldots \ldots \ldots$ & 3.1 & 32 \\
$24.9 \ldots \ldots \ldots$ & 3.5 & 40 \\
\hline
\end{tabular}

bins of $\left.\mathrm{LF}_{V}(\mathrm{~F} 1)\right]$ would not change the global trend of the LF in the regions $\mathrm{F} 1$ and $\mathrm{F} 2$ (given the number of cluster stars in these two fields).

\section{ARTIFICIAL STAR EXPERIMENTS}

Completeness is a stringent requirement for a meaningful astrophysical use of the LF. Therefore, a detailed description of the procedure followed to evaluate the crowding corrections at the faint levels is in order.

An obvious strategy for estimating the fraction of stars lost by DAOPHOT is to add a given number of artificial stars of known magnitude and position to the original image and to ask DAOPHOT to recover them (taking care to follow the same steps and to use the same parameters). The ratio of the number of recovered stars to that of added stars gives the completeness factor $\gamma^{-1}$. Independent experiments were made with stars of fixed magnitude (sampled at steps of 0.3 mag), scattered randomly over the frame. In order to improve the statistics, three experiments per magnitude level were performed, with from 20 to 50 stars added each time.

Obviously, the local crowding must not be altered significantly in the test frames. Therefore, the number of added stars was limited to $2 \%$ of the number $N_{0}$ of measured stars at bright levels, and to $7 \%$ at most at the faintest ones.

Since the most accurate LF is extracted from the c-m diagram ( $\S$ III), a completeness factor of both the $B$ and the $V$ photometry must be provided. For this reason, in each experiment the artificial stars had the same position in the $B$ and $V$ images, with $B-V$ matching the MS value for the given $V$ magnitude.

The typical number of experiments per color was $\sim 30$. The simulations are extremely time-consuming, although they can be completely automatized.

\section{INTERNAL ERRORS}

Artificial star experiments allow us to estimate the internal errors of our photometry as a function of the magnitude level. The internal errors provided by DAOPHOT are usually smaller than the standard deviation of the differences, $\Delta B$ and $\Delta V$, between the added and found magnitudes of the artificial stars (Stetson and Harris 1988). This discrepancy is probably caused by crowding. Tables 6 and 7 give the results of the tests on region F1. Clearly, stars tend to be measured as too bright at all magnitude levels. This may become important at faint magnitudes, where some stars are shifted to brighter levels. The consequence of this "instrumental" redistribution of some faint stars into brighter bins is that the observed LF appears less steep. However, this effect, which is different for different bins and different images, becomes important only at $V>23.0$ (region F1) and $V>22.5$ (F2) for the luminosity

TABLE 6

Crowding Experiments: Region F1, $V$ BAND

\begin{tabular}{|c|c|c|c|c|c|c|c|}
\hline \multicolumn{4}{|c|}{ РнотомeTRY } & \multicolumn{4}{|c|}{ COORdinates } \\
\hline $\boldsymbol{V}$ & $100 / \gamma$ & $\langle\Delta V\rangle$ & $\sigma(\Delta V)$ & $\langle\Delta X\rangle$ & $\sigma(\Delta X)$ & $\langle\Delta Y\rangle$ & $\sigma(\Delta Y)$ \\
\hline $22.47 \ldots \ldots$ & $98 \pm 2.0$ & 0.001 & 0.063 & 0.007 & 0.021 & 0.000 & 0.021 \\
\hline $22.77 \ldots \ldots$ & $70 \pm 1.1$ & -0.001 & 0.097 & -0.002 & 0.034 & 0.000 & 0.031 \\
\hline $23.07 \ldots \ldots$ & $93 \pm 3.1$ & -0.024 & 0.107 & -0.003 & 0.046 & 0.000 & 0.034 \\
\hline $23.37 \ldots \ldots$ & $97 \pm 1.8$ & 0.011 & 0.115 & 0.008 & 0.053 & -0.007 & 0.045 \\
\hline $23.67 \ldots \ldots$ & $94 \pm 2.5$ & -0.028 & 0.157 & 0.007 & 0.062 & -0.010 & 0.051 \\
\hline $23.97 \ldots \ldots$ & $87 \pm 3.0$ & 0.022 & 0.230 & -0.014 & 0.096 & 0.022 & 0.071 \\
\hline $24.27 \ldots \ldots$ & $63 \pm 4.4$ & 0.058 & 0.305 & -0.002 & 0.114 & 0.003 & 0.088 \\
\hline $24.57 \ldots \ldots$ & $44 \pm 4.9$ & 0.160 & 0.452 & 0.007 & 0.116 & 0.004 & 0.117 \\
\hline $24.87 \ldots \ldots$ & $24 \pm 3.9$ & 0.153 & 0.383 & -0.004 & 0.128 & -0.032 & 0.113 \\
\hline
\end{tabular}


TABLE 7

CROWDing EXPERIMENTS: Region F1, B BAND

\begin{tabular}{|c|c|c|c|c|c|c|c|}
\hline \multicolumn{4}{|c|}{ РнотомеTRY } & \multicolumn{4}{|c|}{ COORDINATES } \\
\hline$B$ & $100 / \gamma$ & $\langle\Delta B\rangle$ & $\sigma(\Delta B)$ & $\langle\Delta X\rangle$ & $\sigma(\Delta X)$ & $\langle\Delta Y\rangle$ & $\sigma(\Delta Y)$ \\
\hline $20.97 \ldots \ldots$ & $100 \pm 0.0$ & 0.000 & 0.039 & -0.002 & 0.024 & -0.003 & 0.027 \\
\hline $21.27 \ldots \ldots$ & $95 \pm 2.9$ & -0.005 & 0.037 & 0.001 & 0.022 & 0.001 & 0.034 \\
\hline $21.57 .$. & $95 \pm 4.7$ & -0.001 & 0.050 & -0.005 & 0.024 & 0.002 & 0.034 \\
\hline $21.87 \ldots$ & $93 \pm 4.0$ & -0.015 & 0.052 & -0.006 & 0.047 & -0.010 & 0.052 \\
\hline $22.17 \ldots \ldots$ & $90 \pm 4.0$ & 0.003 & 0.131 & 0.010 & 0.068 & -0.014 & 0.063 \\
\hline $22.47 \ldots$ & $90 \pm 4.3$ & 0.008 & 0.108 & 0.000 & 0.070 & 0.013 & 0.096 \\
\hline $22.77 \ldots$ & $81 \pm 4.9$ & 0.033 & 0.216 & 0.024 & 0.110 & 0.035 & 0.104 \\
\hline $23.07 \ldots \ldots$ & $56 \pm 5.1$ & 0.036 & 0.187 & 0.006 & 0.136 & -0.007 & 0.130 \\
\hline $23.37 \ldots \ldots$ & $28 \pm 4.6$ & 0.099 & 0.203 & 0.010 & 0.113 & -0.008 & 0.120 \\
\hline $23.67 \ldots \ldots$ & $27 \pm 4.5$ & 0.191 & 0.257 & -0.012 & 0.172 & -0.043 & 0.162 \\
\hline $23.97 \ldots \ldots$ & $9 \pm 2.9$ & 0.473 & 0.406 & 0.063 & 0.174 & 0.087 & 0.156 \\
\hline
\end{tabular}

functions derived from the c-m diagram ( $\$$ III), and at $V>24.3$ (F1) and $V>23$ (F2) for the LFs extracted from $V$ photometry, i.e., where completeness is already too low for any meaningful use of the data (Table 3 ).

Our crowding experiments allow us to estimate also the accuracy of DAOPHOT in measuring the stellar centers, in view of possible applications to a proper-motion campaign. The input coordinates of the artificial stars, $X_{\text {in }}$ and $Y_{\text {in }}$, are compared with those provided by the centering algorithm of the reduction package, $X_{\text {out }}$ and $Y_{\text {out }}$. By analyzing the differences $\Delta X\left(=X X_{\text {in }}-X_{\text {out }}\right)$ and $\Delta Y$, we find that, even in a relatively crowded field, the standard errors in each coordinate, $\sigma(\Delta X)$ or $\sigma(\Delta Y)$, are $\lesssim 0$ ". 04 down to $V \simeq 23$ (see cols. [9] and [12] in Table 6).

\section{COMPLETENESS CORRECTIONS}

The final results of the crowding experiments on the two fields F1 and F2 are presented in Figure 8.

The completeness factors for the $V$ and $B$ photometry are comparable in the case of the more crowded region F2. The correction factors for the LF obtained from the $V$ photometry were computed by a smooth interpolation of the output data of the numerical tests.
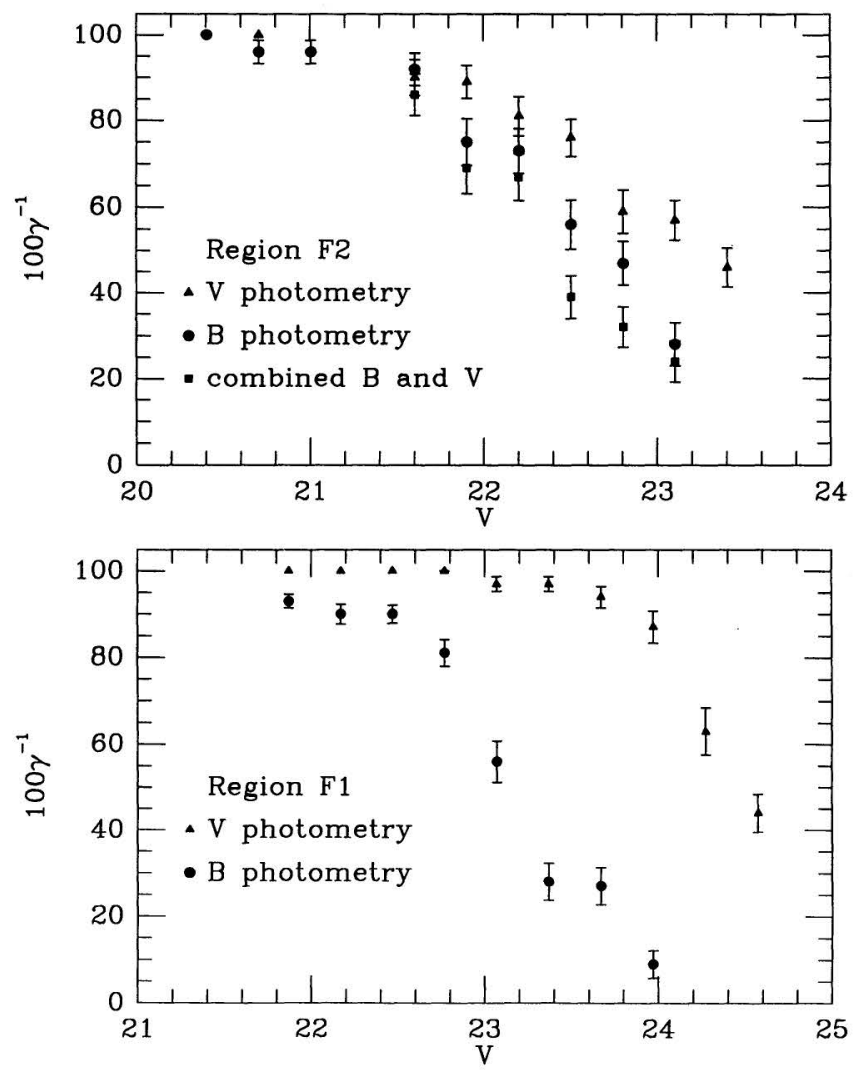

FIG. 8.- Results of crowding experiments, showing the ratio $\gamma^{-1}$ between the number of artificial stars recovered by DAOPHOT and the number added to each frame. Error bars result from comparing three independent experiments. 
The case of the LF from the c-m diagram presents further problems because, for $V>20.5$, stars are lost in the $V$ as well as the $B$ frames. However, it is probable that most of the stars lost in the $V$ are also lost in the $B$ photometry (which is less complete), so it would be incorrect to calculate the completeness factor as the product of the factors for the two photometries. Here we take advantage of the fact that each artificial star had the same position in both colors. The objects recovered by DAOPHOT were cross-identified through their "observed" coordinates in each color and through the input data. For each test, we were able to determine how many artificial stars were found in both colors. This number gives the completeness factor for the LF from the c-m diagram.

Region F1 is less crowded; moreover, the seeing of the $V$ frames was significantly better than that of the $B$ frames. Consequently, the incompleteness of the $B$ image is dominant; e.g., $96 \%$ of the stars were found on the $V$ image at $V=23.5$, while only $\sim 25 \%$ were found on the $B$ image. The completeness factor of the LF from the c-m diagram is thus practically the same as that coming from the tests on the $B$ image.

We may demonstrate now that no significant overcrowding has been introduced by our experiments. Each point in Figure 8 corresponds to three independent crowding experiments; in one of these, we added only half of the stars added in each of the other two. The completeness factor is the same, within the errors. A further check is given by the cross-correlation of the list of stars identified on the artificial images against that of the uncontaminated frames. No "real" star has been lost down to the limiting magnitude significant for the LF.

Bahcall, J. N., and Soneira, R. M. 1980, Ap. J. Suppl., 44, 73.

Bolte, M. 1987, Ap. J., 319, 760 (Bolte).

Capaccioli, M., Ortolani, S., and Piotto, G. P. 1987, Stellar Evolution and Dynamics in the Outer Halo of the Galaxy, ed. M. Azzopardi and F. Matteucci (ESO Conf. Workshop Proc. No. 27), p. 393.

Cordoni, J. P., and Aurière, M. 1984, Astr. Ap. Suppl., 58, 559.

Dickens, R. J. 1972, M.N.R.A.S., 157, 299.

Djorgovski, S., and King, I. R. 1984, Ap. J. (Letters), 277, L49.

Drukier, G. A., Fahlman, G. G., Richer, H. B., and VandenBerg, D. A. 1988, A.J., 95, 1415.

King, I. R., Walterbos, R., Piotto, G. P., Buser, R., and Kurucz, R. 1989, in preparation.

Landolt, A. U. 1973, A.J., 78, 959. $1983, A, J, 88,439$

McClure, R. D., et al. 1986, Ap. J. (Letters), 307, L49.

McClure, R. D., Stetson, P. B., Hesser, J. E., and Smith, G. H. 1987a, in IAU Symposium 126, Globular Cluster Systems in Galaxies, ed. J. Grindlay and A. G. Davis Philip (Dordrecht: Reidel), p. 485.

McClure, R. D., VandenBerg, D. A., Bell, R. A., Hesser, J. E., and Stetson, P. B. $1987 b$, A.J., 93, 1144.

Metcalfe, N., Fong, R., Jones, L. R., and Shanks, T. 1989, in High Redshift and Primeval Galaxies, ed. J. Bergeron, D. Kunth, B. Rocca-Volmerange, and J. Tran Thanh Van (Paris: Editions Frontières), p. 37.

\section{REFERENCES}

Ortolani, S., Piotto, G. P., and Capaccioli, M. 1989, Messenger, 56, 54.

Peterson, C. J., and King, I. R. 1975, A.J., 80, 427.

Piotto, G. P., Capaccioli, M., Ortolani, S., Rosino, L., Alcaino, G., and Liller, W. 1987, A.J., 94, 360.

Piotto, G. P., King, I. R., and Djorgovski, S. 1988, A.J., 96, 1918.

Piotto, 1989 , in preparation.

Pryor, C., Smith, G. H., and McClure, R. D. 1986, A.J., 92, 1358.

Ratnatunga, K. U., and Bahcall, J. N. 1985, Ap. J. Suppl., 59, 63.

Renzini, A., and Fusi Pecci, F. 1988, Ann. Rev. Astr. Ap., 26, 199.

Richer, H. B., Fahlman, G. G., and VandenBerg, D. A. 1988, Ap. J., 329, 187 (RFV).

Seitzer, P., Peterson, R., and Cudworth, K. 1989, in Dynamics of Dense Stellar Systems, ed. D. Merritt (Cambridge: Cambridge University Press), p. 153.

Stetson, P. B. 1987, Pub. A.S.P., 99, 191.

Stetson, P. B., and Harris, W. E. 1988, A.J., 96, 909.

VandenBerg, D. A., and Bell, R. A. 1985, Ap. J. Suppl., 58, 561.

Webbink, R. F. 1985, in IAU Symposium 113, Dynamics of Star Clusters, ed. J. Goodman and P. Hut (Dordrecht: Reidel), p. 541

Weisz, L., and Bell, R. A. 1989, Observatory, 109, 1.

Massimo Capaccioli and Sergio Ortolani: Osservatorio Astronomico, Vicolo dell' Osservatorio, 5, I-35122 Padova, Italy

S. DJoRgovski: Astronomy Department, 105-24 California Institute of Technology, Pasadena, CA 91125

IVAN R. KING: Astronomy Department, University of California, Berkeley, CA 94720

Giampaolo Piotto: Dipartimento di Astronomia, Università di Padova, Vicolo dell' Osservatorio, 5, I-35122 Padova, Italy 